\title{
The effect of bitter, umami and sweet tastants on the food intake regulation
}

\author{
L.S.M.M. Keulers \\ Maastricht University \\ Department: Internal Medicine \\ Ismm.keulers@student.maastrichtuniversity.nl
}

\section{Preface}

After eleven weeks of internship at the department of internal medicine at Maastricht University, this is the summary of my bachelor thesis. It marks the end the marble+ program at the Faculty of Health, Medicine and Life Sciences in Maastricht. Due to intellectual properties this summary only contains a introduction/ background and material and method section.

\section{Abstract}

Background. In the past decades, the prevalence of obesity and overweight has risen to epidemic dimensions. Nowadays, there is a great demand for new and efficient treatments to decrease this prevalence. Literature revealed, that the gastrointestinal tract contains taste receptors, similar to those present on the tongue, which may have an influence on the regulation of food intake and energy expenditure. However the exact working mechanism is unknown.

Objective. This study investigated, the effects of the bitter-, sweet-, umami tastants and a combination of those on ad libitum food intake and gastrointestinal hormone release. Hypothesis. We hypothesized that an intraduodenal infusion of the bitter, umami and sweet tastants and a combination of these tastants, results in a significant reduction in food intake and an significant increase in the release of the gastrointestinal peptides GLP-1 and CCK. Furthermore, the largest reduction on ad libitum food intake, will be achieved by the combination treatment, because this will stimulate multiple taste receptors instead of just one group. Finally, a bitter infusion will induce greater effects on the ad libitum food intake and gastrointestinal hormone release, compared to the sweet and umami tastants. This because the bitter receptor is more sensitive to sense its tastant compared to the others. 
Methods. In this double blind, randomized, placebo controlled, cross-over study, we tested 15 healthy volunteers (Men: 5; Age $23.94 \pm 1.97$ years; BMI $22.43 \pm 0.33 \mathrm{~kg} / \mathrm{m}^{2}$ ). The study consisted of five identical test days. During these days, the effects of the tastants umami (Monosodium glutamate), sweet (Rebaudioside A), bitter (Quinine), a combination of these tastants and the placebo (tap water) treatment, were collected. This by infusing them into the duodenum by means of a nasoduodenal tube. Furthermore, multiple blood samples were drawn before, during and after the infusions to determine gastrointestinal peptide release of GLP-1 and CCK into the blood. Finally, an ad libitum pasta meal was provided to define the effects of the tastants on ad libitum meal intake.

\section{Keywords}

Taste receptors, Bitter, Sweet, Umami, Gastro intestinal tract, Food intake, GLP-1, CCK

\section{Introduction}

In the past decades, the prevalence of obesity and overweight has risen to epidemic dimensions and is now seen as the fifth leading risk among global deaths. According to the World Health Organization, the worldwide obesity rate has nearly doubled since 1980. This resulted in a prevalence of 1.4 billion overweighed adults in 2008, of which 200 million men and 300 million women were obese(1). The increased body weight is associated with several metabolic complications and diseases and has therefore an increased morbidity and mortality rate. Furthermore, obese and overweight individuals also have to face with personal, societal and economic consequences(1-3). All these complications resulted in an increase in research to develop new therapies to treat obesity or prevent the obesity development.

Thus far, bariatric surgery is often used to reduce weight in morbidly obese patients(4). According to Perry et al. these procedures are the only treatment strategies, which have proven to result in long-term weight loss and maintenance in these obese subjects. However these procedures have several disadvantages, such as high costs, high morbidity and mortality rates. Furthermore, this approach can only be applied to a specific group of morbidly obese patients with a BMI above 35 , which have one or more co-morbidities such as hypertension and type 2 diabetes. However, obese individuals with a BMI above 40 do not necessarily need to suffer from co-morbidities to get a surgery $(2,4)$.

Since bariatric surgery can only be used for a specific target group, other approaches are used to treat the obese and overweighed patients with a BMI beneath 40 without these co-morbidities. One of these treatments are the lifestyle modification interventions. These are focused on reducing the caloric intake and increasing energy expenditure, resulting 
in a decrease of the energy imbalance(1). However, long-term studies have shown, that these interventions have poor results and mostly end up with a weight regain(5). This regain in weight is caused by an acute alternation of the hormones, which are involved in the homeostatic regulation of food intake and energy expenditure. These changes in hormone levels include a significant reduction in anorexigenic hormones and a significant increase in orexigenic hormones(6). Furthermore, Leibel et al. demonstrated that weight loss induced a significant decrease of energy expenditure and as a consequences lower the energy requirement. This could also be a cause for the weight regain in lifestyle modification treatments $(7,8)$.

The high costs, mortality and morbidity rates of bariatric surgery and the fact that only a subset of patients can benefit from these therapies show the importance for the development of new efficient therapies in the battle against obesity. Furthermore, the low effectiveness of lifestyle modifications in overweight subjects also demonstrate the great demand for new treatment modalities. For these reasons, a necessity to unravel the homeostatic and hormonal regulation of appetite and satiety has garnered significant attention(2).

\section{Satiety, satiation and the homeostatic food intake regulation}

As explained in the previous paragraph, it is common knowledge that food intake and physical activity regulation are the two main factors involved in body weight maintenance. An important feature to regulate food intake and thereby energy intake, is to alter appetite $(2,9)$. Two main concepts of appetite are satiety and satiation. Satiety is the status that determines the meal intervals and frequency, by inhibiting or reducing the early postprandial meal intake. Satiation refers to the action, that will terminate or reduce the process of eating a meal and is the acute sensation associated with this $(9,10)$.

Satiety and satiation processes, result from a coordinated and integrated neural and hormonal mechanism in response to mechanical and chemical stimuli induced by food intake(9). The neural mechanism is partly caused by distention of the stomach, which is accompanied with food intake. This volume load distention induces a neural signaling by the vagal nerve to the hypothalamus and will eventually lead to a reduction of food intake and hunger(10). However, when food has passed the stomach and enters the duodenum, it will interact with the intestinal wall and induce an increase in neurotransmitters and as a consequence lead to an increasing vagal afferent firing. This will also result in a decreased food intake and an increased satiation and satiety. Besides this increase in nerve reactions, the gut will also synthesize and release several peptide hormones in endocrine cells when nutrients interact with the gut wall $(2,10)$.

These gut peptide hormones, can influence appetite and have an important role 
in the initiation and the termination of a meal(2). Examples of these hormones are cholecystokinin (CCK) and glucagon-like peptide-1 (GLP-1). Both are short-term gut hormones, which play a role in appetite regulation $(2,3)$. Both hormones have anorexigenic effects when they bind to their receptors $(2,3)$. CCK induces a delay in gastric empting and a decrease in gastric acid secretion. Moreover, it will indirectly induce an increase in the pancreatic enzyme secretion and gall bladder contraction $(2,10)$. Besides some of these effects, GLP-1 will induce neuro-hormonal pathways, reduce food intake, and enhance satiety and for this reason, promote weight $\operatorname{loss}(2,3)$. Moreover it has an incretin effect by stimulating the glucose-dependent insulin secretion, promoting the pancreatic $\beta$-cell growth and decreasing the glucagon-secretion, and therefore improving the glycaemic control $(3,10)$.

The above mentioned hormones are two examples of gastrointestinal hormones, which are released in response to food intake. However there are more hormones released before, during and after food intake, and influence the food intake regulation, such as Peptide Tyrosine Tyrosine (PYY), and ghrelin, which is released from the stomach $(2,3,10)$. All these signals, together with the signals of the above mentioned hormones and the stretch receptors, can stimulate the neuronal populations in the hypothalamus or act on the hindbrain, which will form an integrated network and are key regulators of appetite(2, 11). They will promote an accurate food intake, satiety and satiation regulation by promoting or decreasing the food intake, meal termination, satiety and satiation(11).

\section{Taste receptors}

The gut peptide hormones, such as CCK and GLP-1, seem to be released when G-protein coupled receptors (GPCRs) in the enteroendocrine cells get activated(9). These GPCRs in the gastrointestinal tract are a diverse collection of proteins, which can be distinguished by the stimuli on which they respond and the intracellular signaling pathway that they activate. A part of the GPCR proteins are clustered in the family $C$ nutrient sensing receptors, of which the taste receptors are the most important for this study. These are present on the enteroendocrine cells and can sense several luminal chemical components through the gustatory system $(9,12)$. Literature reveales, that this system can sense the five basic perceptions of taste, which are; sweet, salty, sour, bitter and umami $(9,13)$. This will help the human body to protect itself against toxins, helps to select the most suitable, palatable nutrients, regulate satiation, thermogenic effects, give a 'reward value' to food and is responsible for initiating responses, which are critical for survival $(9,12,14,15)$.

The taste receptors are frequently found in taste buds on the tongue, clustered as sweetresponsive heterodimers of T1r2/T1r3, umami-responsive heterodimers of T1r1/T1r3 and 
bitter response receptors, which are called $\operatorname{T} 2 r(9,12,14)$. Several of these taste receptors are expressed in the human solitary epithelial enteroendocrine cells of the gastrointestinal tract and that the distribution of the different taste receptor differ between the duodenum, jejunum, ileum and the colon(14). In contrast to the taste receptors on the tongue, these receptors do not induce taste sensations, but potentially promote the digestion or rejection of food substances through the intestine(16). One of the examples, by which tastants induce this rejection is by the tastant bitter, which is associated with a possible ingestion of harmful toxins $(13,15)$.

Moreover Literature revealed that, when the receptors on the enteroendocrine cells in the gastrointestinal tract are activated by luminal content, this may induce an increase in intracellular $\mathrm{Ca}^{2+}$ level and subsequently result in a release of peptide hormones, such as PYY, GLP-1 and CCK. This may demonstrate an association between satiety and tastants (9, 13). However the effects of the bitter, sweet and umami tastants on this mechanism are contradicting.

\section{Effect of bitter tastants}

Geraedts et al. demonstrated that stimulation of STC-1 cells with the bitter tasting agents quinine and denatonium benzoate (DB), induce a dose-dependent GLP-1 and CCK secretion, of which the GLP-1 secretion increases during the first 30 minutes and the CCK secretion gradually rise during the incubation(13). The same was demonstrated by Chen and Wu et al. which both showed that stimulation of STC-1 cells with DB or phenylthiocarbamide resulted in an increased CCK release $(9,12,15)$.

A possible mechanism for the release of these hormones is that, bitter stimuli activate the bitter taste receptor T2r. This will subsequently promote the synthesis of second messengers, which will induce the intracellular release of $\mathrm{Ca}^{2+}$ and modulate the activation of the L- type voltage-sensitive $\mathrm{Ca}^{2+}$ channels, which in turn mediate the extracellular $\mathrm{Ca}^{2+}$ influx. Both intracellular as well as extracellular $\mathrm{Ca}^{2+}$ will subsequently induce a release of gut peptide hormones $(12,15)$. These can have paracrine or autocrine effects, which include returning the unwanted toxins and substances into the gut lumen or sending signals to the brain to terminate the food intake, reduce gut motility and delay gastric empting(12, $15,16)$. However, the exact mechanism is not clear (16).

\section{Effects of umami tastants}

Umami is described as a 'savoury' flavour and is a key factor, which is often used to improve the taste quality of food $(13,17,18)$. Monosodium glutamate (MSG) simulates this sensory experience. However its effect on satiation and satiety is unclear and has many contradicting research results(19). 
Rogers et al. demonstrated that because of caloric content, MSG will induce a high satiation and fullness feeling after ingestion. However the immediate food intake was uneffected. Furthermore, because of it pleasurable effect, the post prandial satiety signals will not last long and the motivation to eat again will recover very fast(18). In addition to these results, Geraedts et al. showed that stimulating STC-1 cells with the MGS induced an increased secretion of CCK, but poorly influenced the GLP-1 secretion(13).

However, Masic et al showed contradicting results; they demonstrated that MSG has a bi-phasic influence in the food intake regulation. First of all, it induces a reduction in the satiation, because it increases hedonic satisfaction of the food. Moreover, MSG also induces an increase of satiety, which is particularly seen when it is co-ingested with proteins(19). Furthermore according to Hosaka et al. MSG improves the early postprandial glycemia, when it is processed in a lipid containing meal in men. This effect could be related to an increased GLP-1 secretion, 30 minutes after the test meal (20).

\section{Effect of sweet tastants}

In the past decades, the consumption of the natural and artificial sweeteners has risen significantly. However, the effects of sweeteners on satiety and hormone release are still unknown(21).

Anton et al. demonstrated that there was no difference in satiety when humans were provided with a high caloric preload of the natural sweetener sucrose or low caloric preloads of the artificial sweeteners Stevia or aspartame. Furthermore they showed that Stevia induced a lower postprandial insulin and glucose level compared to a sucrose preload. This because of the reduced caloric intake with a Stevia compared to sucrose preload(21).

Another study performed by Geraedts et al. demonstrated that stimulating STC-1 cell with sucrose resulted in a large effect on the CCK secretion, which was followed by the stimulation with sucralose. Moreover, they concluded that CCK release was caloric load dependent and that GLP-1 release was triggered by taste. Finally they also demonstrated that an artificial, non-caloric sweeteners stimuli, resulted in a higher CCK release compared to natural sweetener sucrose. However there were some differences between the different artificial sweeteners on the GLP-1 release, which could be caused by the difference in the composition of these sweeteners(13). 


\section{Research proposal}

As demonstrated in the previous paragraphs, there are many contradicting results about the influence of the tastants on food intake regulation. Furthermore, literature demonstrates, that the taste receptors are down regulated in obese diabetics $(9,17)$. Moreover, there are less to no data available about the complex and multifactorial effects of tastants investigated in a in vivo study; when tastants are injected directly into the duodenum(9). For these reasons, this study investigated, the effects of the tastants bitter (Quinine), sweet (Rebaudioside A) and umami (Monosodium glutamate) and the combination of these three tastants on ad libitum food intake in normal weighed humans. For this, the effect of duodenal delivery of the bitter, sweet and umami tastants or a combination of those on the gastrointestinal hormone release of GLP-1 and CCK, was also investigated. This was subsequently followed by the comparison of the effect of the combination of tastants with the effect of the individual tastants on the ad libitum food intake and gastrointestinal hormone release in the gut. To do this, all tastants were infused into the duodenal lumen by means of a nasoduodenal feeding catheter. By using this application, the taste receptors of the tongue were bypassed. This was not applicable for the prior performed human studies, which often delivered the tastants orally(18). Moreover with this application, the tastant effect may be less influenced by other physiological factors, such as gastric acid, which maybe could induce digestion or deactivation of tastants. Furthermore, the tastants will not be diluted by body fluids, such as salvia and gastric acid. Another advantage of directly infusing the tastants in the duodenum, is that this part of the gastrointestinal tract contains a high amount of taste receptors and as a consequence may induce a significant effect on the investigated parameters, when they are stimulated(14). Therefore, we hypothesized that an intraduodenal infusion of the bitter, umami and sweet tastants and a combination of these tastants, results in a significant reduction in food intake and a significant increase in the release of the gastrointestinal peptides GLP-1 and CCK. Furthermore, the greatest reduction on ad libitum food intake, will be achieved by the combination treatments, because these will stimulate multiple taste receptors instead of just one group. Finally, Depoorte et al. demonstrated that the sweet and umami tastants have a low affinity for their receptors and bitter a high affinity. For this reason, we expect that a bitter infusion will induce greater effects on the ad libitum food intake and gastrointestinal hormone release, compared to the sweet and umami tastants. 


\section{Materials and Methods}

\section{Participants}

A total of 17 healthy human volunteers with a BMI value between 18 and $25 \mathrm{~kg} / \mathrm{m}^{2}$ and an age between 18 and 65 years, participated in this double blind, randomized, placebo controlled, cross over study. However two participants did not finished all the experiments due to discomfort of the catheter.

The subjects were recruited by the website www.digi-prik.nl and posters at the local libraries or other student frequented areas(22). This procedure was followed by receiving verbally and written information in the form of a brochure. Subsequently, before the interested participants were included in the study, they were informed about the study and underwent a medical screening. In this screening, food questionnaires were completed to exclude non-tasters of sweet, bitter and umami tastants. Furthermore, a physical examination was performed to check the heath status and disease history.

Subjects with a history of severe gastrointestinal complications or subjects who underwent a major abdominal surgery interfering with the gastrointestinal function, were excluded from participation in this study. Moreover, individuals were also excluded from participation if they had participated in any scientific intervention study 180 days prior to the study, if they had an administration of investigational drugs, a weight below $60 \mathrm{~kg}$ or if there was evidence of MSG-hypersensitivity.

Besides the abovementioned age and BMI as inclusion criteria, all the participants reported to have no gastrointestinal complaints and to be weight stable for a minimum of six months. Furthermore the women were using contraceptives to avoid a pregnancy.

\section{Study outline}

This double blind, randomized, placebo controlled, cross over study consisted five identical test days, in which five different treatments were investigated by infusing them directly into the duodenum:Treatment 1) Placebo (tap water); Treatment 2) Bitter tastant (quinine); Treatment 3) Umami tastant (Monosodium Glutamate); Treatment 4) Sweet tastant (Rebaudioside A) and Treatment 5) A combinations of the three tastants.

A minimum of two wash-out days were planned between two test days, to avoid carry over effects of the different infusions. However, the order in which the data of the different infusion were collected, were randomly determined by means the Research randomizer, www.Randomizer.org(23). An example of a possible study design is demonstrated in table 1. All together, this study took 12 weeks to complete, in which the individual test day were planned within a period of four weeks. 
Table 1. Example of a study design

\begin{tabular}{l|l}
\hline Day & Infusion \\
\hline Test day 1 & Quinine infusion \\
\hline 2 days wash-out & Rebaudioside A infusion \\
\hline Test day 2 & \\
\hline 2 days wash-out & MSG infusion \\
\hline Test day 3 & \\
\hline 2 days wash-out & Combination infusion \\
\hline Test day 4 & \\
\hline 2 days wash-out & Placebo infusion \\
\hline Test day 5 & \\
\hline
\end{tabular}

\section{Experimental design}

At the start of each of the five test days, a nasoduodenal feeding catheter was placed into the duodenum. Positioning of the tube was followed by the placement of a intravenous cannula in an antecubital vein on the forearm. This was used to collect blood samples of 8 $\mathrm{ml}$, for each time blood was drawn. This blood was sampled to examine the effects of the five treatment on the release of gastrointestinal peptide hormones, such as GLP-1 and CCK. Table 2 demonstrates a scheme of a test day.

Table 2. Test day design

\begin{tabular}{|c|c|c|c|}
\hline Time (min) & $\mathrm{VAS}+\mathrm{GI}$ & Blood samples & Other \\
\hline-45 & & & Arrival at hospital Insertion of catheter \\
\hline-15 & $x$ & $x$ & \\
\hline 0 & & & Liquid breakfast meal \\
\hline 30 & $x$ & $x$ & \\
\hline 90 & $x$ & $x$ & \\
\hline \multicolumn{4}{|l|}{120} \\
\hline 150 & $x$ & $x$ & Start infusion of placebo or tastants \\
\hline 165 & $x$ & $x$ & \\
\hline 180 & $x$ & $x$ & \\
\hline 195 & $\mathrm{x}$ & $x$ & \\
\hline 210 & $x$ & $x$ & End infusion \\
\hline 225 & & & Ad libitum meal \\
\hline 240 & $x$ & $x$ & Test day finished \\
\hline
\end{tabular}


Table 2 shows that after the placement of the catheter and the cannula, the test day starts with the oral ingestion of a standard liquid breakfast. This is considered to be $t=0$ min. After this breakfast, blood samples were collected at regular intervals. 150 minutes after the liquid breakfast ingestion one of the five treatments were infused into the catheter. Moreover, during the infusion period blood samples were collected. These 60 minutes of infusion were subsequently followed by removing the intraduodenal catheter. Fifteen minutes after ending the infusion participants received an oral ad libitum pasta meal to investigate the ad libitum food intake. Participants were instructed to eat as much as they desired.

\section{Catheter positioning}

In this study, a Bengmark catheter was placed transnasally into the duodenum. First, we used a local anaesthetic to anaesthetize the nasal mucosa and placed the catheter via the nose into the stomach. Second, an intermittent fluoroscopic control was used to place the catheter tip into the duodenum. The latter was performed at the radiology department of MUMC+ after an eight hour fast. Furthermore, the participants were not allowed to consume alcohol and participating in exhaustive physical exercise one day prior the test day. Finally at the end of the test day, the catheter was removed.

\section{Gastrointestinal peptides}

To investigate the effect of the different tastants on the release of gastrointestinal peptide hormones, such as CCK and GLP-1, the levels of the collected blood samples at the onset of the infusion until the end of the ad libitum meal were analyzed and compared to the results of the baseline measurements.

We used a chilled aprotinin-coated tube, which was used for the CCK and GLP-1 data collection. After the blood collection in this tube, dipeptidyl peptidase IV inhibitor was immediately added, to prevent degradation of GLP-1. This step was followed by centrifuging and the storage of the samples.

Finally the GLP-1 levels were assessed by using a Glucagon Like Peptide-1 ELISA kit and CCK levels were determined by using a radioimmunoassay.

\section{Statistical analyses}

To analyze the collected data, we used SPSS statistical software package. To check for normality, a histogram was drawn, which demonstrated the normality curve. When this curve showed a normal distribution of the data, this was checked with a Shapiro-Wilk test, in which a p-value $<0,05$ demonstrated a non-parametric distribution. When the data 
turned out not to be normal distributed, a log transformation was used to normalize the data. Eventually when the variables were normally distributed, the data were analyzed by calculating the area under the curve (AUC) by means of the trapezoid method. All data were analyzed by a linear mixed model to investigate if there was a difference in the effect between the treatments. When there was a significant difference ( $p$-value<0,05), a Bonferroni correction for multiple testing was applied. Furthermore, when there was a significant difference in effects according to the analysis of the AUCs, the whole dataset was analyzed by a linear mixed model, to check on which time points these treatments significantly differed.

\section{Endnote}

Obesity and overweight are now seen as the fifth leading risk among global deaths. Both have to face with several metabolic complications and have personal, societal and economic consequences. All these complications and consequences resulted in an increasing interest to develop new therapies to treat obesity or prevent its development. Literature revealed, that the gastrointestinal tract contains taste receptors, which may have an influence on the regulation of food intake and energy expenditure. For this reason we investigated the effects of the bitter-, sweet-, umami tastants and a combination of those on ad libitum food intake and gastrointestinal hormone release of CCK en GLP-1. We hypothesized that the results of this study could be a onset for further research to develop an anti-obesity treatment by the use of tastants. Unfortunately, due to intellectual properties no results are demonstrated. However, when there are some questions or when you are interested, contact me on the following address Ismm.keulers@student.maastrichtuniversity.nl

\section{References}

1. WHO. Obesity and overweight [updated May 2014]. Available from: http://www.who.int/mediacentre/ factsheets/fs311/en/.

2. Perry B, Wang Y. Appetite regulation and weight control: the role of gut hormones. Nutrition \& diabetes. 2012;2:e26.

3. Suzuki K, Jayasena CN, Bloom SR. The gut hormones in appetite regulation. Journal of obesity. 2011;2011:528401.

4. Heelkunde Nvv. Richtlijn Morbide Obesitas 2011. Available from: http://www.mdl.nl/uploads/240/1000/ Richtlijn-morbide-obesitas-final.pdf.

5. Weigle DS, Breen PA, Matthys CC, Callahan HS, Meeuws KE, Burden VR, et al. A high-protein diet induces sustained reductions in appetite, ad libitum caloric intake, and body weight despite compensatory changes in diurnal plasma leptin and ghrelin concentrations. The American journal of clinical nutrition. 2005;82(1):41-8.

6. Sumithran P, Prendergast LA, Delbridge E, Purcell K, Shulkes A, Kriketos A, et al. Long-term persistence of hormonal adaptations to weight loss. The New England journal of medicine. 2011;365(17):1597-604.

7. Rosenbaum M, Hirsch J, Gallagher DA, Leibel RL. Long-term persistence of adaptive thermogenesis in subjects who have maintained a reduced body weight. The American journal of clinical nutrition. 2008;88(4):906-12. 
8. Leibel RL, Hirsch J. Diminished energy requirements in reduced-obese patients. Metabolism: clinical and experimental. 1984;33(2):164-70.

9. Geraedts MC, Troost FJ, Saris WH. Gastrointestinal targets to modulate satiety and food intake. Obesity reviews : an official journal of the International Association for the Study of Obesity. 2011;12(6):470-7.

10. Maljaars J, Peters HP, Masclee AM. Review article: The gastrointestinal tract: neuroendocrine regulation of satiety and food intake. Alimentary pharmacology \& therapeutics. 2007;26 Suppl 2:241-50.

11. Foster-Schubert KE, Cummings DE. Emerging therapeutic strategies for obesity. Endocrine reviews. 2006;27(7):779-93.

12. Chen MC, Wu SV, Reeve JR, Jr., Rozengurt E. Bitter stimuli induce Ca2+ signaling and CCK release in enteroendocrine STC-1 cells: role of L-type voltage-sensitive Ca2+ channels. American journal of physiology Cell physiology. 2006;291(4):C726-39.

13. Geraedts M. C. P. TFJ, Saris W. H. M. . Different tastants and low-caloric sweeteners induce differential effects on the release of satiety hormones. Food Chemistry. 2011;129:731-8.

14. Bezencon C, le Coutre J, Damak S. Taste-signaling proteins are coexpressed in solitary intestinal epithelial cells. Chemical senses. 2007;32(1):41-9.

15. Wu SV, Rozengurt N, Yang M, Young SH, Sinnett-Smith J, Rozengurt E. Expression of bitter taste receptors of the T2R family in the gastrointestinal tract and enteroendocrine STC-1 cells. Proceedings of the National Academy of Sciences of the United States of America. 2002;99(4):2392-7.

16. Finger TE KS. Taste isn't just for taste buds anymore. F100o Biol Rep2011 [updated 2014]. Available from: http:// www.the-scientist.com/?articles.view/articleNo/31441/title/Matters-of-Taste/.

17. Ruiz CJ, Wray K, Delay E, Margolskee RF, Kinnamon SC. Behavioral evidence for a role of alpha-gustducin in glutamate taste. Chemical senses. 2003;28(7):573-9.

18. Rogers PJ, Blundell JE. Umami and appetite: effects of monosodium glutamate on hunger and food intake in human subjects. Physiology \& behavior. 1990;48(6):801-4

19. Masic U, Yeomans MR. Does monosodium glutamate interact with macronutrient composition to influence subsequent appetite? Physiology \& behavior. 2013;116-117:23-9.

20. Hosaka H, Kusano M, Zai H, Kawada A, Kuribayashi S, Shimoyama Y, et al. Monosodium glutamate stimulates secretion of glucagon-like peptide-1 and reduces postprandial glucose after a lipid-containing meal. Alimentary pharmacology \& therapeutics. 2012;36(9):895-903.

21. Anton SD, Martin CK, Han H, Coulon S, Cefalu WT, Geiselman P, et al. Effects of stevia, aspartame, and sucrose on food intake, satiety, and postprandial glucose and insulin levels. Appetite. 2010;55(1):37-43.

22. Delta T. Digi-prik 2012. Available from: http://www.digi-prik.nl/.

23. Urbaniak G, \& Plous, S Research Randomizer (Version 4.0) 2013 [updated June 22th, 2013]. Available from: http://www.randomizer.org/. 